\title{
Segurança Alimentar e Nutricional: avaliação e fatores determinantes em consórcio de municípios, Bahia, Brasil
}

\author{
Food and Nutritional Security: evaluation and determining factors in cities consortium, \\ Bahia, Brazil
}

Joelma Cláudia Silveira Ribeiro'

Sandra Maria Chaves dos Santos²

Tânia Mara Buranelli Soares ${ }^{3}$

Juçara Ana Bastos da Costa Accioly ${ }^{4}$

' Força Aérea Brasileira - FAB (da Base Aérea de Salvador-BA). Salvador, BA, Brasil.

${ }^{2}$ Universidade Federal da Bahia, Escola de Nutrição, Departamento de Ciências da Nutrição. Salvador, BA, Brasil.

${ }^{3}$ Secretaria Municipal da Saúde SMS. Salvador, BA, Brasil.

${ }^{4}$ Universidade Federal da Bahia, Centro de Nutrição e Políticas Públicas. Salvador, BA, Brasil.

Este projeto faz parte do Projeto de extensão intitulado Segurança Alimentar no Vale do Jiquiriçá - BA: Bases para Construção Participativa dos Sistemas Locais. Aprovado no edital 038/2008 MDS/CNPQ, tem por objetivo geral subsidiar a organização dos sistemas locais de SANdo edital 038 - Josué de Castro, visando promover ações de extensão no campo da SAN em CONSADs.

Correspondência / Correspondence

Joelma Cláudia Silveira Ribeiro

E-mail: caunuti@hotmail.com

\section{Resumo}

Introdução: $\mathrm{O}$ marco legal brasileiro para a promoção e garantia da segurança alimentar e nutricional contemplou a implementação de um sistema. O desafio de construir este sistema de Segurança Alimentar e Nutricional municipal envolve informações e conhecimento da situação e seus fatores determinantes, que geralmente estão deficientes ou inexistem em municípios menores. Estudos nessa direção orientam a gestão local para intervenções de maior impacto na alteração das condições de vida da população. Nessa perspectiva, foram criados os Consórcios de Segurança Alimentar e Desenvolvimento Local para o enfrentamento dos problemas sociais. Objetivo: avaliar a situação de segurança alimentar e seus determinantes em municípios integrantes do Consórcio. Método: Utilizaram-se a Escala Brasileira de Insegurança Alimentar, questionário socioeconômico e protocolo de indicadores para caracterizar a situação de segurança alimentar e nutricional. Resultados: A Escala Brasileira demonstrou insegurança alimentar em 70,3\% dos domicílios, com predomínio da insegurança grave e moderada $(36,0 \%)$. O protocolo de indicadores identificou insegurança alimentar leve nas dimensões de disponibilidade, consumo e utilização biológica de nutrientes e insegurança moderada para acesso, sendo possível indicar quais variáveis estão definindo a situação. Conclusão: Os instrumentos permitiram obter os resultados que indicam existir déficits sociais que comprometem a segurança alimentar das famílias. Estes podem orientar a gestão para ações e intervenções que minimizem esta situação, sendo subsídios estratégicos no planejamento, elaboração, 
monitoramento de políticas públicas locais na promoção do Direito Humano à Alimentação Adequada.

Palavras-chave: Segurança Alimentar e Nutricional. Indicadores Sociais. Política Pública.

\section{Abstract}

Introduction: The Brazilian legal mark for the promotion and guarantee of Feeding and Nutritional Security contemplated the implementation of a system. The challenge of building this municipal Food and Nutrition Security System involves information and knowledge of the situation and its determining factors, that are generally deficient or do not exist in smaller municipalities. Studies in this direction guide the local management for interventions that have greater impact on changing the population's living conditions. From this perspective, the Consortia of Feeding Security and Local Development were created to deal with social problems. Objective: To evaluate the feeding security situation and its determinants in municipalities that part of the Consortium. Method: The Brazilian Scale of Food Insecurity, a socioeconomic questionnaire and the indicators protocol were used to characterize the food and nutritional security situation. Results: the Brazilian Scale showed food insecurity in $70.3 \%$ of households, with predominance of severe and moderate insecurity (36.0\%). The indicators protocol identified light food insecurity in the dimensions of availability, consumption and biological use of nutrients and moderate insecurity for access, indicating which variables are defining the situation. Conclusion: The instruments made it possible to obtain the results that show there are social deficits that compromise the families' food security. These can guide the management to actions and interventions that minimize this situation, being strategic subsidies in the planning, elaboration, monitoring of local public policies for the Human Right to Adequate Feeding promotion.

Keywords: Food and Nutritional Security. Social Indicators. Public Policy. 


\section{Introdução}

A Segurança Alimentar e Nutricional (SAN) constitui um objetivo estratégico e permanente de políticas públicas orientadas pelos princípios do Direito Humano à Alimentação adequada e da Soberania Alimentar, que engloba e qualifica a erradicação da fome, da desnutrição e manifestações mais graves de insegurança alimentar. ${ }^{1}$ É compreendida como a garantia, a todos, de condições de acesso a alimentos básicos de qualidade, em quantidade suficiente, de modo permanente e sem comprometer o acesso a outras necessidades básicas, com base em práticas alimentares que possibilitem a saudável reprodução do organismo humano, contribuindo para uma existência digna. ${ }^{2}$ Quando ocorre a violação a esse direito humano, são geradas situações de insegurança alimentar. ${ }^{3}$

A Organização das Nações Unidas para Agricultura e Alimentação (FAO) orienta e recomenda que a SAN seja promovida desde o município, como possível organizador de ações políticas. $\mathrm{O}$ Brasil fundamentou este princípio com a homologação da Lei n.․․ 11.346, que instituiu o Sistema Nacional de SAN (SISAN), tornando-se um desafio sua construção em âmbitos nacional, estadual e municipal.

Apesar da realização de pesquisas nacionais para avaliação da SAN domiciliar no país, um dos obstáculos que ocorrem para implantar e implementar sistemas de SAN é a deficiente informação sobre a situação e seus determinantes em municípios mais pobres e de menor porte, exatamente onde se pressupõe haver maior risco de insegurança alimentar e nutricional, a exemplo dos resultados da Pesquisa Nacional de Amostra Domiciliar (PNAD)., ${ }^{4,5}$

No Brasil, observa-se o investimento no tratamento de questões sociais na perspectiva territorial; isto é, há uma tendência de serem analisadas e dirigidas intervenções não somente para indivíduos ou famílias. Assim, foram criados os Consórcios de Segurança Alimentar e Desenvolvimento Local (CONSADs), objetivando promover a articulação entre municípios de baixo índice de desenvolvimento humano, resultantes da parceria entre poder público e sociedade civil. Estes integram ações e programas gerenciados pelo Ministério do Desenvolvimento Social e Combate à Fome (MDS).

Este artigo apresenta resultados da análise de fatores relacionados com a ocorrência de insegurança alimentar em municípios que integram o CONSAD Vale do Jiquiriçá, entendendo ser tal conhecimento estratégico para intervenções consequentes e potencialmente capazes de reduzir o problema no território. 


\section{Método}

\section{Cenário do Estudo}

A criação de consórcios no Brasil segue na direção de valorizar o espaço onde vivem os sujeitos e viabilizar ações conjuntas. Tem como objetivo maior tornar permanente as articulações para a promoção de ações de forma institucionalizada, visando prioritariamente integrar as esferas da produção, comercialização, consumo e crédito nas regiões mais pobres do país que enfrentam maior risco de insegurança alimentar e nutricional. Trata-se, assim, de uma modalidade eficiente de cooperação que possibilita discutir, planejar, decidir e implantar ações de desenvolvimento local e melhoria de vida das populações envolvidas. ${ }^{6}$

Segundo dados do Ministério de Desenvolvimento Social e Combate à Fome, estão implantados no Brasil 40 CONSADs, contemplando todas as regióes do país. Na Bahia foram implantados dois: o de Brumado, na região sudoeste, e o do Vale do Jiquiriçá, no Recôncavo Baiano. Por questões logísticas e tendo em vista experiências acumuladas em pesquisa e extensão na região, foi selecionado o CONSAD do Vale do Jiquiriçá para desenvolvimento deste estudo. Ele foi implantado em 2003, no bojo do Programa Fome Zero, com a finalidade de coordenar a implantação das ações e projetos agroalimentares nos nove municípios do Vale que integram a área deste consórcio.

O Vale do Jiquiriçá, localizado a cerca de $160 \mathrm{~km}$ da capital, é formado por 25 municípios de médio e pequeno porte, dos quais somente cinco superam 20 mil habitantes e conformam o Território de Identidade do Vale do Jiquiriçá, forma de organização territorial adotada pelo Estado da Bahia para implementar políticas públicas. ${ }^{7}$ Segundo a Cooperativa Nacional de Assessoria e Planejamento (CONAP) para implantação do CONSAD, o associativismo pode ser considerado bom, pois contemplaria várias associações de produtores, associações comunitárias, sindicatos profissionais, cooperativas de produção e de crédito da região. A agricultura familiar predomina na exploração diversificada de culturas de subsistência, como mandioca, feijão, milho e banana. ${ }^{8}$

Os municípios do Vale, em geral, possuem baixo grau de desenvolvimento, principalmente se consideradas as informações médias para o Estado da Bahia, mostrando a relevância do desenvolvimento de projetos que possam colaborar para conhecer os problemas e assim intervir de forma efetiva e eficaz. ${ }^{8}$

A pesquisa foi aprovada pelo Comitê de Ética em Pesquisa da Escola de Nutrição da Universidade Federal da Bahia (ENUFBA), sob parecer 207.389. Todos os participantes assinaram um Termo de Consentimento Livre e Esclarecido (TCLE). 


\section{Trabalho de campo}

\section{Aplicação da Escala Brasileira de Segururança Alimentar (EBIA)}

Para o estudo domiciliar por meio da avaliação direta, foi adotada a técnica da amostragem aleatória estratificada, sendo cada município dividido em dois estratos: área urbana e área rural. O tamanho da amostra de cada estrato foi calculado adotando-se o critério pelo qual se mantém a fração de amostragem em cada estrato igual à fração global de amostragem com alocação sistemática, ou ainda, adotando a partilha proporcional.

O tamanho calculado da amostra para este estudo foi de 2.591 domicílios, para os municípios com precisão de $5 \%$ com prevalência de $50 \%$ de insegurança alimentar e nutricional (ISAN Moderada e Grave) para as zonas rural e urbana. Partiu-se inicialmente do número de domicílios segundo dados do censo do Instituto Brasileiro de Geografia e Estatística (IBGE). Após, considerouse o número de domicílios e aplicou-se a regra de proporcionalidade em cada município, obtendose o número de domicílios a serem visitados em cada localidade para dar conta da amostragem.

Os agentes comunitários de saúde (ACS) foram mobilizados e treinados para a coleta de dados, considerando que eles alcançam $100 \%$ dos domicílios na região, conhecem detalhadamente as áreas urbanas e rurais, são reconhecidos e respeitados pela população, tendo em vista que, entre suas ações, está a orientação das várias políticas públicas no campo da saúde. ${ }^{9}$ As entrevistas realizadas favoreceram a capacitação de atores locais.

Em cada localidade, os ACS foram orientados a contar e numerar o total de domicílios, calcular o intervalo sistemático (total de domicílios dividido pelo número de domicílios a serem visitados) e a realizar um sorteio para definir o início aleatório.

Para a avaliação direta, os dados foram coletados por meio de visitas domiciliares no período de novembro de 2010 a fevereiro de 2011, com aplicação de dois instrumentos - um para avaliar a segurança/insegurança alimentar, através da Escala Brasileira de Insegurança Alimentar; e outro, o questionário para dados sociais e econômicos dos moradores, para obter fatores explicativos para os achados.

A Escala Brasileira de Insegurança Alimentar utilizada é uma versão adaptada e validada daquela elaborada pelo Departamento de Agricultura dos Estados Unidos (United States Department of Agriculture - USDA). Trata-se de um instrumento que tem sido aplicado em plano nacional, com o propósito de medir, diretamente, a percepção de insegurança alimentar e fome em nível domiciliar, o que possibilita a estimativa de sua prevalência na população. ${ }^{10}$

O questionário foi aplicado para a caracterização social e econômica das famílias investigadas, pois o mesmo contempla múltiplas informações, considerando variáveis que têm mostrado maior associação com a insegurança alimentar em outros estudos. 
Para este estudo, foram consideradas as seguintes variáveis: Renda familiar mensal (considerouse como referência o salário-mínimo vigente (01/01/2010 - R \$ 510,00), sendo categorizada em três estratos: < 1/4 salário-mínimo; 1/4 a 1/2 salários-mínimos e > 1/2 salário-mínimo; Escolaridade do chefe do domicílio (considerados três níveis conforme as séries escolares cursadas: I - até a $4^{\mathrm{a}}$ série; II - da 5 $5^{\mathrm{a}}$ a $8^{\mathrm{a}}$ série; e, III - Ensino médio e superior). Sexo do responsável pelo domicílio (masculino ou feminino). Para moradia familiar, foram analisadas características de: Saneamento básico (acesso a rede de abastecimento de água, acesso a coleta de lixo, existência de esgotamento sanitário). O índice foi classificado em dois estratos: adequado (escore $\leq 04$ ), quando as respostas foram positivas para todos os itens, e inadequado (escore $>04$ ), quando a resposta foi negativa para pelo menos um dos itens; Número de habitantes por domicílio (até 4 habitantes, 4-6 habitantes e $\geq 7$ habitantes); Acesso a programas sociais: foram verificadas informações sobre acesso das pessoas do domicílio a programas sociais de diferentes tipos e sob responsabilidade de diferentes esferas de governo, e também de organizações não governamentais.

\section{Avaliação da SAN Municipal}

O protocolo de indicadores adotado neste estudo foi desenvolvido a partir da seleção e criação de indicadores considerados capazes de expressar a situação de municípios de pequeno porte, não alcançados em geral por pesquisas nacionais que aplicam a EBIA e sem capacidade técnica e financeira para realizar estudos específicos. ${ }^{11}$ Detalhes desse protocolo, a exemplo do nome dos indicadores nas diferentes dimensões, os parâmetros e a pontuação (entre 0 e 10), os meios de verificação e as premissas que permitem dar inteligibilidade aos resultados obtidos, estão dispostos no quadro 1. Para leitura dos resultados, assumiu-se a escala dividida em quatro partes, de tal forma que a soma dos resultados por dimensão pode corresponder a percentuais diferentes de desempenho nas dimensões, as quais se referem a diferentes situações de segurança alimentar e nutricional e insegurança alimentar e nutricional, conforme apresentado no quadro 1. 
Quadro 1. Escala de valores e categorias para avaliação da segurança alimentar e nutricional municipal com uso de um protocolo de indicadores. Bahia, 2008.

\begin{tabular}{|c|c|c|}
\hline Percentual dos pontos & Categoria & Definição \\
\hline$>75$ & $\begin{array}{l}\text { Segurança } \\
\text { alimentar e } \\
\text { nutricional }\end{array}$ & $\begin{array}{l}\text { Representa uma situação ideal em que } \\
\text { um conjunto de fatores presentes nas } \\
\text { dimensões considerada atuaria para } \\
\text { garantir a realização plena do conceito de } \\
\text { segurança alimentar e nutricional }\end{array}$ \\
\hline 50 a 74,9 & $\begin{array}{l}\text { Insegurança } \\
\text { alimentar e } \\
\text { nutricional leve }\end{array}$ & $\begin{array}{l}\text { Representa uma situação em que } \\
\text { alguns fatores, presentes nas dimensões } \\
\text { consideradas comprometeriam a } \\
\text { realização plena do conceito, com relativa } \\
\text { vulnerabilidade à privação e à fome }\end{array}$ \\
\hline 25 a 49,9 & $\begin{array}{l}\text { Insegurança } \\
\text { alimentar e } \\
\text { nutricional } \\
\text { moderada }\end{array}$ & $\begin{array}{l}\text { Representa uma situação em que um maior } \\
\text { número de fatores, presentes nas dimensões } \\
\text { consideradas, comprometeria a realização } \\
\text { plena do conceito, caracterizando uma } \\
\text { situação de maior vulnerabilidade à } \\
\text { privação e à fome. }\end{array}$ \\
\hline 0 a 24,9 & $\begin{array}{l}\text { Insegurança } \\
\text { alimentar e } \\
\text { nutricional grave }\end{array}$ & $\begin{array}{l}\text { Representa uma situação em que um maior } \\
\text { número de fatores, presentes nas dimensões } \\
\text { consideradas, comprometeria a realização } \\
\text { plena do conceito, caracterizando uma } \\
\text { situação de privação e fome }\end{array}$ \\
\hline
\end{tabular}

Fonte: Panelli-Martins et al. (2008).

O instrumento por objetivo aperfeiçoar uma metodologia para avaliação em municípios, por meio de indicadores calculados a partir de dados secundários disponíveis em bases de dados públicas, como o Departamento de Informática do Sistema Único de Saúde (DATASUS), IBGE, Sistema de Informação da Atenção Básica (SIAB), entre outros. A plataforma do site é uma ferramenta para registrar dados e conhecer a situação de segurança alimentar e nutricional da população municipal. A avaliação é realizada por um conjunto de indicadores, e os gestores podem obter o diagnóstico da 
situação considerando as dimensões da disponibilidade de alimentos, acesso aos alimentos, consumo de alimentos e utilização biológica de nutrientes, conforme definidas a seguir:

- Disponibilidade de alimentos - relacionada a aspectos vinculados a produção e comercialização local de alimentos, em quantidade suficiente e de modo permanente;

- Acesso aos alimentos - refere-se a aspectos socioeconômicos e físicos que interferem na aquisição de alimentos pela população;

- Consumo de alimentos - relaciona-se ao padrão alimentar de uma população e aos fatores sociais, econômicos e culturais que interferem no mesmo;

- Utilização biológica de nutrientes - inclui as condições de acesso a serviços sociais, de saneamento e de saúde que, ao incidirem sobre o estado de saúde do indivíduo, podem limitar a utilização biológica dos nutrientes. ${ }^{11}$

\section{Análise dos Dados}

Iniciou-se a avaliação direta domiciliar com análise descritiva para medir a frequência das variáveis em estudo. Em seguida, fez-se a associação entre as variáveis através do teste quiquadrado, análise gráfica e técnicas inferenciais pertinentes. Para medir a associação entre a variável-desfecho e as independentes, foram definidas três categorias de desfecho: Segurança Alimentar, Insegurança Alimentar Leve, Insegurança Alimentar Moderada/Grave - esta última tratada como categoria de referência.

Foram realizadas a análise de regressão logística politômica univariada e a técnica de regressão logística politômica multivariada. Permaneceram no modelo apenas aquelas variáveis com valor de $p<0,05$. As informações foram transcritas para meio digital, com checagem de inconsistência e amplitudes, sendo utilizado o Programa Epi Info 6.04 (Centers for Disese Controland Prevention Atlanta - Estados Unidos) por dupla digitação. As análises estatísticas foram desenvolvidas com auxílio da plataforma estatística Statistical Packadge Social of Sciences (SPSS) para Windows (versão 13.0).

\section{Resultados}

\section{A SAN domiciliar}

Na aplicação da EBIA, verificou-se que a insegurança alimentar atinge 70,3\% dos domicílios no CONSAD (Figura 1). 


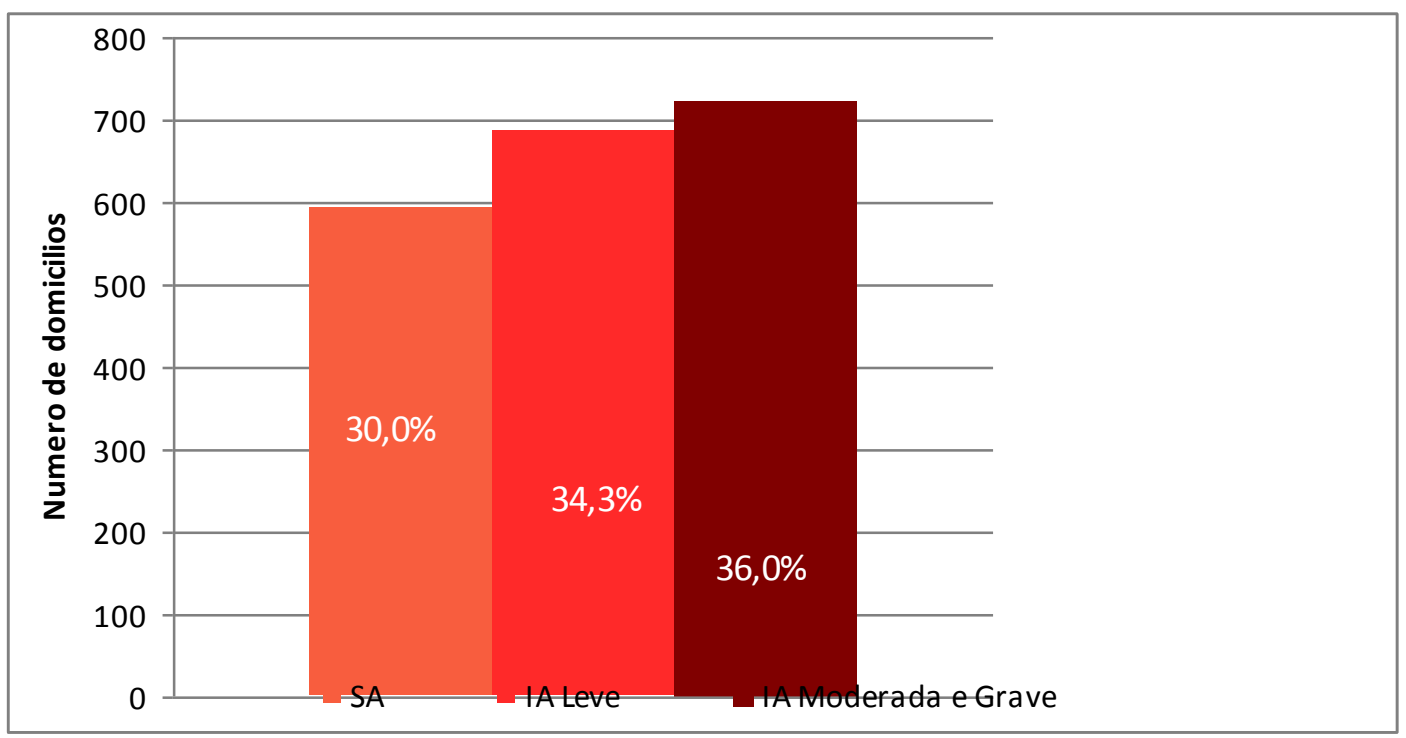

Figura 1. Percentual de domicílios em situação de insegurança alimentar. CONSAD Vale do Jiquiriçá-BA, 2011

Fonte: Levantamento domiciliar, 2010-2011.

Os resultados da análise de regressão logística politômica, adotada no estudo para identificar as associações de interesse na elucidação da dimensão e distribuição da insegurança alimentar no território estudado, são verificados na tabela 1 .

A chefia da família exercida por mulheres predominou nos domicílios investigados, mas não houve associação positiva e estatisticamente significante entre o sexo da pessoa de referência (feminino) e insegurança alimentar moderada e grave. Tal relação, no entanto, se apresentou para a insegurança alimentar leve. Observou-se associação estatisticamente significante entre escolaridade e insegurança alimentar leve (categoria "até 4⿳亠丷厂 série de estudos") e insegurança alimentar moderada/grave (categoria "até 4⿳亠丷a série de estudos" /IC 95\%) e categoria " 5 a a 8ª série de estudos".

A Insegurança Alimentar Leve, Moderada/Grave mostrou associação positiva e estatisticamente significante (IC 95\%) para as variáveis: escolaridade pelo responsável do domicílio, condições de vida no domicílio em relação a renda familiar per capita menor de 1⁄4 do salário-mínimo, em relação ao maior número de moradores por domicílio e para o indicador ambiental também mostrou associação positiva e significante. No entanto para presença de morador menor de 18 anos no domicílio a associação foi observada apenas para Insegurança Alimentar Leve. 
Tabela 1. Associação entre insegurança alimentar e variáveis demográficas e socioeconômicas. Vale do Jiquiriçá-BA, 2011.

\begin{tabular}{|c|c|c|c|c|}
\hline Variáveis & $\mathrm{n}$ & $\%$ IA Leve & $\begin{array}{c}\% \text { IA Moderada } \\
\text { e Grave }\end{array}$ & $\begin{array}{l}\text { Valor } \\
\text { de p }\end{array}$ \\
\hline \multicolumn{5}{|l|}{ Sexo do responsável * } \\
\hline Masculino & 369 & 29,5 & 35,8 & 0,119 \\
\hline Feminino & 931 & 33,4 & 37,6 & \\
\hline \multicolumn{5}{|l|}{ Renda per capita } \\
\hline$<1 / 4$ Salário-mínimo & 997 & 32,2 & 46,5 & $<0,001$ \\
\hline$\geq 1 / 4$ Salário-mínimo & 647 & 35,9 & 18,1 & \\
\hline \multicolumn{5}{|c|}{ Morador menor de 18 anos } \\
\hline Sim & 1799 & 33,3 & 39,6 & $<0,001$ \\
\hline Não & 203 & 43,3 & 3,9 & \\
\hline \multicolumn{5}{|c|}{ Escolaridade do responsável } \\
\hline Até a $4^{\mathrm{a}}$ série & 872 & 30,5 & 41,6 & $<0,001$ \\
\hline $5^{\underline{a}}$ a $8^{\underline{a}}$ série & 157 & 38,2 & 42,7 & \\
\hline Ensino médio e mais & 259 & 33,6 & 19,3 & \\
\hline \multicolumn{5}{|l|}{$\begin{array}{l}\text { Número de moradores no } \\
\text { domicílio }\end{array}$} \\
\hline Até 3 pessoas & 732 & 34,8 & 25,8 & $<0,001$ \\
\hline 4 a 6 pessoas & 966 & 34,6 & 38,8 & \\
\hline$\geq 7$ pessoas & 150 & 21,3 & 65,3 & \\
\hline \multicolumn{5}{|l|}{ Indicador ambiental } \\
\hline Inadequado & 1083 & 32,8 & 40,5 & $<0,001$ \\
\hline Adequado & 778 & 34,8 & 29,6 & \\
\hline \multicolumn{5}{|l|}{ Zona de residência } \\
\hline Rural & 1166 & 33,4 & 39,0 & 0,003 \\
\hline Urbana & 836 & 35,5 & 31,8 & \\
\hline \multicolumn{5}{|l|}{ Programa Bolsa Família } \\
\hline Sim & 1122 & 33,1 & 45,9 & $<0,001$ \\
\hline Não & 519 & 31,8 & 23,1 & \\
\hline
\end{tabular}

Fonte: Levantamento domiciliar, 2010-2011. 


\section{A SAN em Âmbito Municipal}

Utilizando o protocolo de indicadores, foi possível identificar para o CONSAD Vale do Jiquiriçá, considerando todas as dimensões um desempenho de 53,7\% da pontuação total, situando-o na faixa de insegurança alimentar e nutricional (ISAN leve), conforme o quadro 2.

Quadro 2. Avaliação da situação de segurança alimentar e nutricional por dimensões no Vale do Jiquiriçá-BA, 2011.

\begin{tabular}{|l|c|c|c|l|}
\hline \multicolumn{1}{|c|}{ Dimensão } & $\begin{array}{c}\text { Pontuação } \\
\text { máxima }\end{array}$ & $\begin{array}{c}\text { Pontuação } \\
\text { obtida }\end{array}$ & $\begin{array}{c}\text { Escala de } \\
\text { pontos }(\%)\end{array}$ & \multicolumn{1}{|c|}{$\begin{array}{c}\text { Situação de } \\
\text { SAN }\end{array}$} \\
\hline $\begin{array}{l}\text { Disponibilidade de } \\
\text { alimentos }\end{array}$ & 60 & 35,0 & 58,3 & IAN leve \\
\hline Acesso aos alimentos & 30 & 14,4 & 48,1 & IAN moderada \\
\hline Consumo de alimentos & 30 & 17,2 & 56,7 & IAN leve \\
\hline $\begin{array}{l}\text { Utilização biológica de } \\
\text { alimentos }\end{array}$ & 60 & 30,0 & 50,0 & IAN leve \\
\hline Total & 180 & 96,6 & 53,7 & ISAN leve \\
\hline
\end{tabular}

Fonte: Ribeiro (2011).

Nas dimensões "Disponibilidade", "Consumo" e "Utilização biológica de alimentos", os indicadores somaram uma pontuação que classificaram como insegurança alimentar leve. No entanto, para a dimensão de "Acesso aos Alimentos", a classificação pontuou para insegurança alimentar moderada.

\section{Discussão}

A prevalência de insegurança alimentar (IA) encontrada no território foi bastante elevada (70,3\% dos domicílios), tendo em vista que resultados de outras pesquisas com a aplicação da EBIA também evidenciaram percentuais de domicílios em insegurança alimentar aquém dos resultados obtidos no território. Em estudos realizados no município de Duque de Caxias, ${ }^{12}$ no Rio de Janeiro, encontrou-se prevalência de 53,8\% de IA em domicílios dessa localidade; na Paraíba, pesquisadores encontraram 52,5\% de insegurança em domicílios de 14 municípios de maior pobreza. ${ }^{13}$ No entanto, estudos realizados em regiões mais pobres alcançaram resultados 
distintos, como em pesquisa desenvolvida (nas zonas urbana e rural) no município de São João do Tigre, microrregião dos Cariris Velhos, Estado da Paraíba, com 458 famílias com menores de 5 anos, em queforam encontrados $87,3 \%$ de insegurança alimentar. ${ }^{14}$

Estudos sobre a distribuição da insegurança alimentar têm apontado para a importância da expansão dos programas sociais brasileiros na redução do problema. No entanto, mesmo com a redução observada para o Nordeste e para a Bahia, áreas específicas desse estado convivem ainda com prevalências altas do problema. A insegurança alimentar não afeta apenas municípios do interior: em Salvador, capital, estudo realizado em 2007 em amostra de domicílios particulares de famílias de escolares da rede pública encontrou prevalência de $71,3 \%$ de insegurança alimentar. ${ }^{15}$

Para compreender melhor os resultados na prevalência, a análise multivariada revelou que fatores estavam aumentando as chances de famílias nos domicílios viverem insegurança alimentar, quais sejam: a renda mensal familiar per capita $<1 / 4$ do salário mínimo; número de moradores no domicílio maior que quatro pessoas; indicador ambiental inadequado. A interpretação desses resultados pode orientar a gestão pública no território sobre a direção das ações necessárias.

Neste estudo, como em outros, a renda mensal familiar per capita se mostrou relevante para a definição da insegurança alimentar. A moderada e grave estava presente em 46,5\% dos domicílios com renda mensal familiar per capita inferior a 1/4 do salário-mínimo (SM). Esta prevalência se reduziu para 24,1\% entre os domicílios com renda entre meio e 1/4 do salário-mínimo, e para 8,4\% entre aqueles com renda mensal maior do que meio SM. Considerando apenas duas categorias de renda, a prevalência de Insegurança Alimentar moderada e grave foi de 46,5\% para aqueles com renda menor que $1 / 4$ do SM, resultado que se mostrou estatisticamente significante. Na análise detalhada, evidenciou-se que domicílios com renda mensal menor de 1/4 do salário-mínimo tinham quase três vezes mais chances de estarem em situação de IA moderada e grave. Em domicílios com renda média familiar per capita menor que $1 / 4$ do SM cuja responsável era do sexo feminino, a IA moderada e grave ficou bem acima daquela registrada no domicílio cujo responsável era do sexo masculino: respectivamente, $21,2 \%$ e $16,7 \%$.

No Brasil, 43,1\% dos domicílios que estavam na classe de rendimento mensal domiciliar per capita de até $1 / 4$ salário-mínimo apresentaram situação de insegurança alimentar. ${ }^{5}$ Estudo realizado por Souza na Região Nordeste observou que, entre os domicílios com renda per capita de até 1/4 do salário-mínimo, a chance de estarem em IA foi 27,3 vezes maior do que para outras faixas de renda. ${ }^{16}$ Estudos conduzidos por diversos autores comprovaram que quanto menor a classe de rendimento mensal domiciliar per capita, maior a proporção de domicílios em situação de IA moderada e grave..$^{13,17}$

Muitas das variáveis consideradas na análise de IA têm relação direta com o número de moradores no domicílio, e esta tem sido uma variável com força explicativa para a insegurança alimentar em vários estudos. Neste estudo, mais de 50,0\% dos domicílios somavam 4-6 moradores, 
e $8,1 \%$ contavam com sete ou mais moradores. A IA moderada e grave se apresentou em $38,8 \%$ dos domicílios com 4-6 moradores e alcançou 65,3\% dos domicílios com sete ou mais moradores. Esses resultados foram estatisticamente significantes (tabela 2). Na análise de chances, os domicílios com sete pessoas ou mais obtiveram 3,19 vezes mais chances (IC95\% 1,80 - 5,68) de a família estar em situação de IA moderada e grave. Esta chance se reduziu para 1,22 vezes (IC95\% 0,90-1,65) entre os domicílios com 4-6 pessoas. Estudo realizado por Anschau encontrou razão de chances 83,0\% maior para IA moderada e grave em domicílios com cinco ou mais moradores. ${ }^{18}$

Tabela 2. Odds ratio ajustada para avaliar os fatores associados à insegurança alimentar e nutricional dos domicílios do CONSAD, Vale do Jiquiriçá-BA.

\begin{tabular}{|c|c|c|c|c|}
\hline \multirow{2}{*}{ Variáveis } & \multicolumn{2}{|c|}{ IA leve } & \multicolumn{2}{|c|}{ IA Moderada/Grave } \\
\hline & OR & IC95\% & OR & IC95\% \\
\hline \multicolumn{5}{|l|}{ Renda per capita } \\
\hline$\geq 1$ 1/4 Salário-mínimo & 1 & - & 1 & - \\
\hline <1/4 Salário-mínimo & 1,45 & $1,070-1,957$ & 2,91 & $2,117-4,000$ \\
\hline \multicolumn{5}{|c|}{ № de moradores no domicilio } \\
\hline Até 3 pessoas & 1 & - & 1 & - \\
\hline 4 a 6 pessoas & 1,11 & $0,829-1,481$ & 1,22 & $0,905-1,651$ \\
\hline$\geq 7$ pessoas & 1,28 & $0,677-2,426$ & 3,19 & $1,796-5,677$ \\
\hline \multicolumn{5}{|l|}{ Indicador ambiental } \\
\hline Adequado & 1 & - & 1 & - \\
\hline Inadequado & 0,95 & $0,722-1,243$ & 1,34 & $1,012-1,771$ \\
\hline \multicolumn{5}{|l|}{ Programa Bolsa Família } \\
\hline Sim & 1 & - & 1 & - \\
\hline Não & 1,81 & $1,325-2,463$ & 2,31 & $1,665-3,205$ \\
\hline
\end{tabular}

Fonte: Levantamento domiciliar, 2010-2011.

As condições de moradia estão intimamente relacionadas com a vulnerabilidade das famílias à IA. Em domicílios com alta densidade populacional por cômodo utilizado para dormir, a IA aparece com maior prevalência. ${ }^{16}$ 
Desde os anos 1990, há o entendimento de que a Segurança Alimentar, além da dimensão alimentar e sanitária, contempla a qualidade sanitária dos alimentos e sua inocuidade, incluindo-se a água. Este aspecto está também contemplado na definição brasileira de SAN, conforme previsto na Lei Orgânica de Segurança Alimentar e Nutricional (LOSAN). ${ }^{2}$

Neste estudo, as características de moradia, considerando o acesso a serviços de saneamento básico (água tratada, existência de sanitário no domicílio e de esgotamento sanitário), foram integradas em um indicador ambiental, dicotômico, atribuindo-se a condição de adequado ou inadequado ao domicílio. Nos domicílios estudados, $58,2 \%$ foram classificados como inadequados e $41,8 \%$ como adequados. A IA se apresentou em 73,3\% dos domicílios com inadequação ambiental, resultado estatisticamente significante. Focando a IA grave e moderada, esta foi registrada em 40,5\% dos domicílios com condições inadequadas e em 29,6\% daqueles considerados adequados - diferencial importante para qualificar a intensidade da IA que não se apresentou para a IA leve. $\mathrm{Na}$ análise de chances, registrou-se que resultados negativos em relação aos serviços indicados aumentavam em 1,34 vezes as chances de IA moderada e grave, sinalizando a importância de corrigir tais problemas.

Os programas de transferência de renda foram criados no Brasil como uma estratégia de complementação de renda em face da situação de desemprego e baixa renda de parcelas significativas da população. No CONSAD Vale do Jiquiriçá, 68,4\% dos domicílios estudados estavam contemplados com o benefício do Programa Bolsa Família (PBF); destes, 45,9\% se encontravam em IA. Esses resultados indicam a focalização adequada do programa naqueles que mais necessitam do benefício e são compatíveis com achados de outras pesquisas. ${ }^{13}$ Do ponto de vista das políticas públicas, o programa é importante para melhorar as condições de vida das famílias, embora, por si só, não garanta índices satisfatórios de segurança alimentar, questão associada a um quadro de pobreza mais amplo. ${ }^{19}$

\section{A SAN no Município}

Resultados identificados por pesquisa utilizando o Protocolo de Indicadores no município de Mutuípe-BA mostraram uma situação de insegurança alimentar e nutricional moderada, considerando as dimensões de disponibilidade, acesso, consumo e utilização biológica. ${ }^{11}$

A Segurança Alimentar e Nutricional na área estudada, na dimensão da disponibilidade de alimentos, está relacionada a aspectos vinculados a produção e comercialização local de alimentos. Isto significa dizer que, para o município, tendo em vista os indicadores utilizados, não existem problemas importantes para o abastecimento alimentar da população. Contribuíram para tais resultados fatores como a existência de: vias de acesso ao município e entre zona rural e urbana em boas condições, facilitando o escoamento dos produtos produzidos, o que pode favorecer maior 
oferta de alimentos como menor custo; estratégias de aproximação do pequeno produtor com consumidor devido à existência de feira livre semanal, facilitando o acesso da população de menor renda aos alimentos; uma cooperativa de pequenos produtores, favorecendo o aumento da oferta local de alimentos e da renda das famílias dos pequenos produtores; diversidade de linhas de crédito ao pequeno produtor, refletindo positivamente na agricultura familiar e no autoconsumo, aumentando a renda e garantindo condições mínimas de manutenção das atividades agropecuárias.

Mesmo tendo obtido bom resultado nesta dimensão, registrou-se no município a inexistência de armazéns que pudessem garantir a estocagem de alimentos para abastecer o mercado interno por pelo menos dois meses. Isso garantiria que possíveis problemas que afetassem a produção, como alterações climáticas e a comercialização, devido à entressafra que eleva os preços, por exemplo, não comprometessem o acesso de toda a população aos alimentos.

Na dimensão de "Acesso de alimentos", o principal fator que contribuiu para o resultado obtido foi a baixa escolaridade do responsável pelo domicílio, avaliada como anos de estudo. Esta influencia não só nas chances de obter melhor remuneração no trabalho, como na seleção, aquisição e preparo dos alimentos, estando também associada à possibilidade de utilização de bens e serviços essenciais à manutenção do estado de saúde. Os mesmos resultados foram encontrados no município de Mutuípe, com aplicação do mesmo instrumento. ${ }^{11}$

Na dimensão "Consumo de alimentos", os fatores que contribuíram para os resultados encontrados foram: alto percentual de crianças com baixo-peso ao nascer, ressaltando-se a relação com o estado nutricional materno durante a gestação interferindo diretamente no estado de saúde e sobrevida da criança; alto percentual de crianças com menos de cinco anos com déficit ponderal para idade, sendo que este indica um estado de desnutrição atual, refletindo más condições de saúde, renda e educação familiar.

O alto percentual de aleitamento materno exclusivo foi um resultado importante do estudo na definição de um quadro de IA leve, partindo-se do pressuposto de que o mesmo fornece nutrientes adequados qualitativa e quantitativamente para o crescimento. O estudo citado identificou que os mesmos indicadores estavam relacionados com resultados similares.

Na dimensão de "Utilização biológica dos alimentos", o resultado obtido se deve à deficiente cobertura do saneamento básico, tais como: falta de acesso a água tratada, coleta de lixo doméstico e esgotamento sanitário, de forma que a exposição familiar a condições adversas de saneamento básico influencia diretamente na saúde da família. O resultado do estudo no município de Mutuípe não foi diferente do resultado do CONSAD. ${ }^{11}$

Importante ressaltar que o coeficiente de mortalidade infantil contribuiu para melhores resultados nesta dimensão, a partir da premissa de que esse indicador reflete as condições de saúde da população em geral. Cabe informar que o Programa Saúde da Família estava implementado e com cobertura de 100\% pelos Agentes Comunitários de Saúde em todos os municípios deste estudo. 


\section{Conclusão}

Na medida em que se colocou na agenda política a criação dos Sistemas Locais de Segurança Alimentar e Nutricional, interessaria aos CONSADs agir neste campo, para modificar as condições de vida das pessoas, na perspectiva territorial, o que exige informações sobre a situação e seus condicionantes. Assim, o presente estudo teve como propósito identificar a situação de segurança alimentar e nutricional e seus fatores determinantes em municípios do território do Consórcio de Segurança Alimentar e Desenvolvimento Local - CONSAD Vale do Jiquiriçá.

Para gerar os resultados e análises necessárias, a pesquisa aplicou a Escala Brasileira de Insegurança Alimentar (EBIA), o questionário socioeconômico e o protocolo de indicadores, para explorar a situação local e os fatores associados aos resultados. Sendo um estudo transversal, e na total ausência de estudos prévios no território sobre o assunto, este trabalho enfrentou fatores limitantes, como a impossibilidade de estabelecer relações causais entre a variável desfecho insegurança alimentar - e as variáveis independentes consideradas.

Em síntese, a análise global dos resultados indica que existem déficits sociais que estão comprometendo a Segurança Alimentar e Nutricional das famílias. Ressalta-se que os problemas se apresentaram menos associados a atributos dos sujeitos que vivem a insegurança, e mais fortemente associados a suas condições sociais dependentes da ação pública. Portanto, o conjunto desses resultados pode orientar gestores para ações e intervenções que são potencialmente capazes de minimizar a insegurança alimentar e ser subsídios estratégicos do planejamento, elaboração, monitoramento de políticas públicas locais, com o objetivo de promover o Direito Humano à Alimentação Adequada.

Os métodos aplicados apresentaram-se como instrumentos factíveis para a avaliação de SAN domiciliar e municipal. A Escala Brasileira de Insegurança Alimentar tem sido muito utilizada em diversos estudos e sobretudo pela Pesquisa Nacional por Amostra Domiciliar. O protocolo de indicadores desenvolvido para aplicação em âmbito local apresentou-se como instrumento importante para avaliação de SAN municipal, considerando que tratou de avaliar a SAN em quatro dimensões, implicando o acesso a dados e informações de diferentes sistemas.

\section{Agradecimentos}

Aos professores e funcionários da Escola de Nutrição da Universidade Federal da Bahia (ENUFBA). À Coordenação de Aperfeiçoamento de Pessoal Ensino de Nível Superior (CAPES), pela concessão da bolsa de mestrado. Ao Conselho Nacional de Desenvolvimento Científico e Tecnológico (CNPq), pela oportunidade de desenvolver este estudo. A toda equipe e bolsistas do Núcleo de Nutrição e Políticas Públicas da Escola de Nutrição da Universidade Federal da Bahia (NNPP-ENUFBA). 


\section{Colaboradores}

Ribeiro JCS participou de todas as etapas da pesquisa, do planejamento e das atividades em campo, da concepção, análise e interpretação dos dados. Santos SMC foi responsável pela coordenação geral do projeto. Buranelli Soares TM e Accioly JABC participaram de todas as etapas da pesquisa.

Conflito de Interesses: Os autores declaram não haver conflitos de interesse.

\section{Referências}

1. Maluf RS. Segurança alimentar e fome no Brasil: 10 anos da cúpula mundial de alimentação. Relatórios técnicos 2. Rio de Janeiro: Ceresan; 2006.

2. Brasil. Lei no 11.346, de 15 de setembro de 2006. Cria o Sistema Nacional de Segurança Alimentar e Nutricional - SISAN com vistas em assegurar o Direito Humano à Alimentação Adequada e dá outras providências. Diário Oficial da União. 18 set. 2006.

3. Gubert MB, Santos LMP. Determinantes da insegurança alimentar no Distrito Federal. Comun Ciências Saúde. 2009; 20(2):143-150.

4. Instituto Brasileiro de Geografia e Estatística. Pesquisa Nacional por Amostra de Domicílio. Segurança alimentar. PNAD 2004 [Internet]. Rio de Janeiro: IBGE; 2006. Disponível em: https://biblioteca.ibge. gov.br/visualizacao/monografias/GEBIS\%20-\%20RJ/segalimentar/suguranca_alimentar2004.pdf

5. Instituto Brasileiro de Geografia e Estatística. Pesquisa Nacional por Amostra de Domicílio. Segurança alimentar 2004/2009 [Internet]. Rio de Janeiro: IBGE; 2010. Disponível em: https://biblioteca.ibge. gov.br/visualizacao/livros/liv47241.pdf

6. Brasil. Ministério do Desenvolvimento Social e Combate à Fome. Segurança Alimentar e Nutricional. Trajetória e Relatos da Construção de uma Política Nacional. Brasília: MDS; 2008.

7. Olalde AR. Dinâmicas territoriais rurais no Vale do Jequiriçá, Bahia, Brasil. VIII Congresso LatinoAmericano de Sociologia Rural; 15-19 nov. 2010; Porto de Galinhas, PE.

8. Bahia. Cooperativa Nacional de Assessoria e Planejamento. Diagnóstico participativo. Consórcios de Segurança Alimentar e Desenvolvimento Local do Vale do Jiquiriçá. Salvador: CONSAD; 2002.

9. Burlandy L. Transferência condicionada de renda e segurança alimentar e nutricional. Ciênc Saúde Coletiva. 2007; 12(6):1441-1451.

10. Kepple AW, Segall-Corrêa AM. Conceituando e medindo segurança alimentar e nutricional. Ciênc Saúde Coletiva. 2011; 16(1):187-199.

11. Panelli-Martins BE. Análise de métodos de avaliação da segurança alimentar e nutricional: uma contribuição à política municipal de SAN [dissertação]. [Salvador]: Universidade Federal da Bahia; 2008.

12. Salles-Costa R, Pereira RA, Vasconcellos MTL, Veiga GV, Marins VMR, Jardim BC, et al. Associação entre fatores sócio-econômicos e insegurança alimentar: estudo de base populacional na Região Metropolitana do Rio de Janeiro, Brasil. Rev Nutrição. 2008; 21(Supl.):S99-S109. 
13. Viana RPT, Segall-Cossêa AM. Insegurança alimentar das famílias residentes em municípios do interior do estado da Paraíba, Brasil. Rev Nutrição. 2008; 219(Supl.):S111-S122.

14. Oliveira JS. Insegurança alimentar e estado nutricional de crianças de São João do Tigre, no semiárido do Nordeste. Rev Bras Epidemiol. 2009. 12(3):413-423.

15. Bittencourt LS. Fatores associados à insegurança Alimentar de famílias de escolares da rede pública de ensino de Salvador, Bahia, Brasil [dissertação]. Salvador: Universidade Federal da Bahia; 2010.

16. Souza LM. Fatores associados à insegurança alimentar nos domicílios da Região Nordeste do Brasil, 2004 [tese]. [Belo Horizonte]: Universidade Federal de Minas Gerais; 2009.

17. Pimentel PG, Sichieri R, Salles-Costa R. Insegurança alimentar, condições sócio-econômicas e indicadores antropométricos em crianças da Região Metropolitana do Rio de Janeiro, Brasil. R Bras Est Pop. 2009; 26(2):283-294.

18. Anschau FR. Insegurança alimentar de beneficiários de programas de transferência de renda [dissertação]. [Londrina]: Universidade Estadual de Londrina; 2008.

19. Instituto Brasileiro de Análises Sociais e Econômicas. Repercussões do Programa Bolsa Família na Segurança Alimentar e Nutricional das famílias beneficiadas. Documento síntese. Rio de Janeiro: IBASE; 2008.

Recebido: 14 de agosto, 2017

Revisado: 30 de janeiro, 2018

Aceito: 05 de março, 2018 